\section{Staphylococcal endogenous endophthalmitis in association with pyogenic vertebral osteomyelitis}

LR Steeples and NP Jones
Manchester Royal Eye Hospital, Manchester, UK

Correspondence: LR Steeples, Manchester Royal Eye Hospital, Oxford Road, Manchester M13 9WL, UK

Tel: 44 (0)161 2761234.

E-mail: laurasteeples@

hotmail.com

Received: 9 May 2015 Accepted in revised form: 8 August 2015 Published online: 9 October 2015

\author{
Abstract \\ Purpose To describe pyogenic vertebral \\ osteomyelitis as a rare infection associated \\ with endogenous endophthalmitis. \\ Methods A retrospective review of three \\ patients with endogenous endophthalmitis \\ and sepsis due to underlying Staphylococcal \\ vertebral osteomyelitis presenting during a \\ 21-month time period. The ophthalmic and \\ systemic features and management and \\ outcomes are presented. \\ Results One patient developed unilateral \\ endophthalmitis with cervical spine \\ osteomyelitis, Staphylococcus aureus being \\ isolated from blood cultures. The second \\ presented with bilateral endophthalmitis with \\ disseminated Methicillin-resistant S. aureus \\ (MRSA) infection, with thoracic and lumbar \\ discitis and para-spinal abscesses. MRSA was \\ cultured from vitreous, blood, and synovial \\ fluid. Both patients received prolonged \\ courses of intravenous antibiotics. Intravitreal \\ antibiotic therapy was used in the second \\ patient. Excellent visual and systemic \\ outcomes were achieved in both cases with \\ no ocular complications. The third patient \\ developed lumbar osteomyelitis following \\ spinal surgery and presented with \\ disseminated $S$. aureus sepsis including \\ unilateral endogenous endophthalmitis. \\ Despite systemic antibiotics and intensive \\ care the patient died. \\ Conclusions Endogenous endophthalmitis \\ should be suspected in septic patients \\ developing eye symptoms. Endogenous \\ endophthalmitis with staphylococcal bone \\ infection is a rare but serious condition. \\ Osteomyelitis should be considered as an \\ infective source in any such patient \\ reporting bone pain or reduced spinal \\ mobility. Prompt investigation and
}

treatment can achieve favourable visual and systemic outcomes.

Eye (2016) 30, 152-155; doi:10.1038/eye.2015.200; published online 9 October 2015

\section{Introduction}

Endogenous bacterial endophthalmitis (EBE) accounts for $2-8 \%$ of all reported cases of endophthalmitis. ${ }^{1}$ It is associated with poor visual outcomes and high mortality rates. ${ }^{2}$ Staphylococcus aureus and Streptococcus species account for most cases in Western countries. ${ }^{1,2}$

In the largest published series of $342 \mathrm{EBE}$ cases, the commonest foci of infection were lung, liver, endocardium, and urinary tract. ${ }^{2}$ However, only $2(0.58 \%)$ occurred in association with osteomyelitis. Spinal osteomyelitis is a highly unusual association, with very few published case reports. ${ }^{3-5}$ All involved Streptococcus species rather than $S$. aureus.

\section{Case reports}

\section{Case 1}

A 71-year-old female presented with floaters and reduced vision in her left eye and a 1-week history of neck pain. The left visual acuity (VA) was $6 / 36$. The left eye had panuveitis with significant vitritis and a focus of retinitis superonasally (Figure 1a). EBE was not initially considered but she subsequently developed pyrexia $\left(38.3^{\circ} \mathrm{C}\right)$ with raised inflammatory markers (ESR $124 \mathrm{~mm} / \mathrm{h}$, CRP $369 \mathrm{mg} / \mathrm{l}$ ). Bacterial sepsis and EBE was now most the probable diagnosis. Empirical intravenous ceftriaxone ( $2 \mathrm{~g}$ daily) was initiated. Blood cultures identified gram-positive cocci. Intravenous teicoplanin (600 mg daily) and 
oral rifampicin (450 mg BD) were added following microbiology advice to cover for probable Staphylococcal infection. Her uveitis improved significantly after $24 \mathrm{~h}$ of treatment. Magnetic resonance imaging (MRI) of cervical spine performed 2 days later showed C3/4 osteomyelitis with a large para-spinal collection and epidural abscess (Figures 2a and b). Methicillin-sensitive Staphylococcus aureus (MSSA) was cultured from blood. Treatment with a cervical collar and a 3-month course of antibiotic therapy was given (Table 1). She made a full recovery with 6/9 acuity.
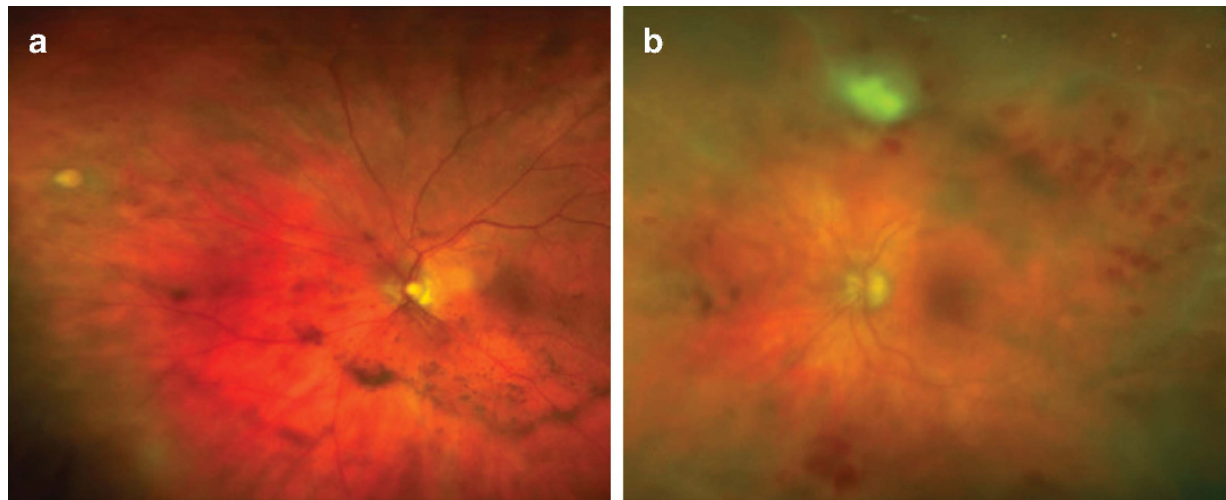

Figure 1 (a) Case 1, fundal photograph of the left eye at presentation with vitreous opacities and a supero-nasal focus of retinitis and surrounding haemorrhage. (b) Case 2, fundal photograph of the left eye at presentation with a focus of retinitis superior to the disc, widespread peripheral vasculitis and patchy retinal haemorrhage.
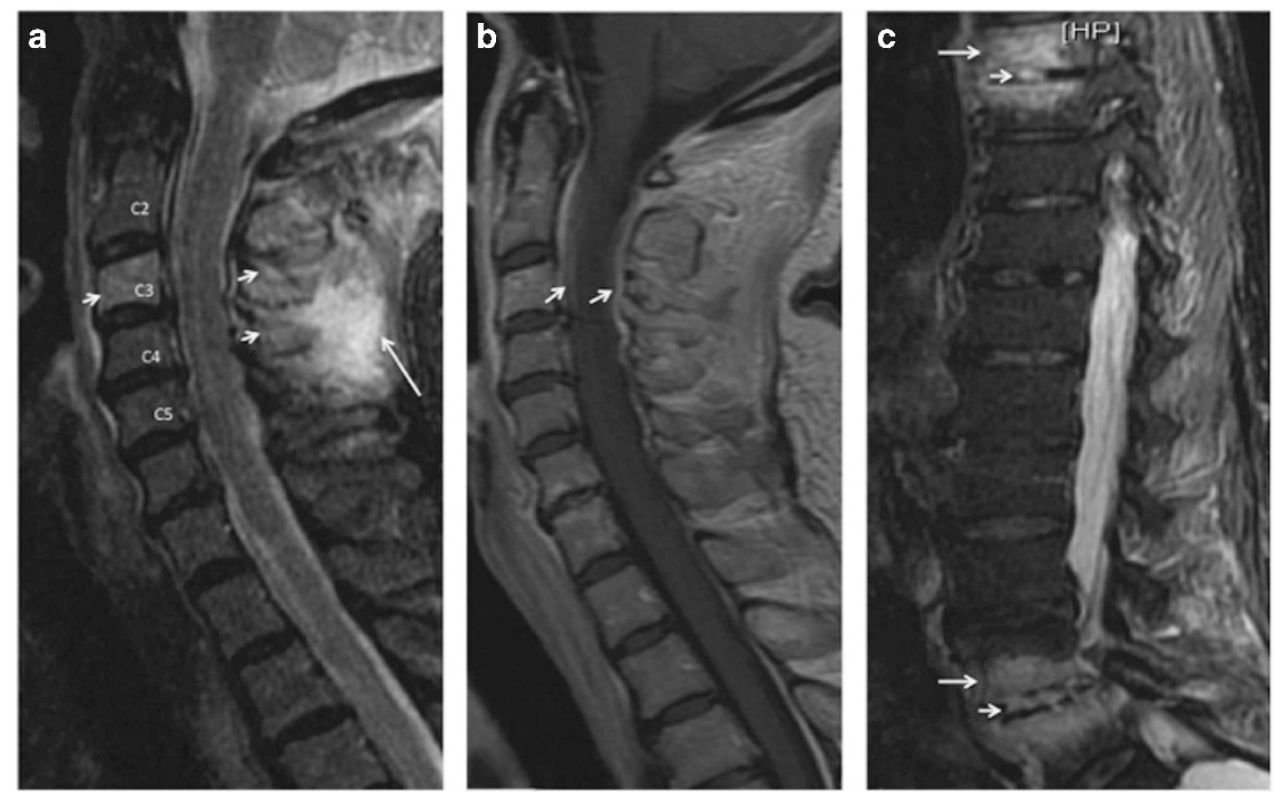

Figure 2 (a) Case 1, sagittal MRI images of the cervical spine demonstrating loculated para-spinal collection, extending from the skull base to C5 and centred on the tissues posterior to C3-C4 (long arrow) with osteomyelitis involving the C3 vertebral body and C3/C4 facet joints (short arrows). (b) Case 1, post-contrast MRI image illustrating epidural enhancement in the C1-C3 region. (c) Case 2, sagittal MRI images illustrating thoracic and lumbar osteomyelitis involving T8/9 and L4/5 vertebral bodies (long arrows) with discitis (short arrows). 
Table 1 Clinical summary of the three cases including presentation and management

\begin{tabular}{|c|c|c|c|}
\hline & Case 1 & Case 2 & Case 3 \\
\hline Predisposing illness & None & None & $\begin{array}{l}\text { Spinal surgery } \\
\text { Former IVDU, HIV negative }\end{array}$ \\
\hline Primary focus of infection & $\begin{array}{l}\text { Cervical spine }(\mathrm{C} 3 / 4) \text { osteomyelitis } \\
\text { with epidural abscess and para- } \\
\text { spinal abscess }\end{array}$ & $\begin{array}{l}\text { Thoracic (T8/9) and lumbar (L4/5) } \\
\text { spine osteomyelitis with discitis and } \\
\text { epidural abscess }\end{array}$ & Lumbar spine osteomyelitis \\
\hline Affected eye, initial VA & L $6 / 36$ & R CF, L 6/36 & $\mathrm{L}$ N/A \\
\hline Ocular classification & L panuveitis with posterior focus & $\begin{array}{l}R \text { vitritis, } L \text { panuveitis with posterior } \\
\text { focus }\end{array}$ & L panuveitis, no fundal view \\
\hline Vitreous culture & Not taken & + & + \\
\hline Blood culture & + & + & + \\
\hline Other culture-positive foci & None & Wrist, urine, and skin & Spinal wound \\
\hline Micro-organism & MSSA & MRSA & MSSA \\
\hline Intravitreal treatment & None & V (2 separate doses) & $\mathrm{V}, \mathrm{C}$ \\
\hline Systemic antibiotic & CTX, T, Ri & V, Ri, D & $\mathrm{T}, \mathrm{Ri}$ \\
\hline Affected eye: final VA & L $6 / 9$ & $\mathrm{R} 6 / 12, \mathrm{~L} 6 / 6$ & $\mathrm{~N} / \mathrm{A}$ \\
\hline Systemic outcome & $\begin{array}{l}\text { No long-term neurological or } \\
\text { musculoskeletal sequelae }\end{array}$ & $\begin{array}{l}\text { Chronic back pain } \\
\text { No long-term neurological sequelae }\end{array}$ & Death \\
\hline Late ocular sequelae & None & None & $\mathrm{N} / \mathrm{A}$ \\
\hline
\end{tabular}

Abbreviations: C, ceftazidime; CTX, ceftriaxone; $\mathrm{CF}$, counting fingers; D, Doxycycline; $\mathrm{F}$, female; HIV, human immunodeficiency virus; IVDU, intravenous drug abuse; L, left; M, male; MRSA, methicillin-resistant Staphylococcal aureus; MSSA, methicillin-sensitive S. aureus; N/A, not available; +, positive; $\mathrm{R}$, right; $\mathrm{Ri}$, rifampicin; $\mathrm{T}$, teicoplanin; $\mathrm{V}$, vancomycin; $\mathrm{VA}$, visual acuity.

Inflammatory markers were elevated (CRP $333 \mathrm{mg} / \mathrm{l}$, ESR $90 \mathrm{~mm} / \mathrm{h}$ ). Intravenous gentamicin and vancomycin and oral rifampicin were started empirically due to severity of presentation, covering for suspected Staphylococcal infection. He underwent left vitreous sampling and intravitreal vancomycin $(1 \mathrm{mg} / 0.1 \mathrm{ml})$ injection. MRSA was isolated from blood, vitreous and multiple extraocular sites (Table 1). MRI revealed thoracic and lumbar spine osteomyelitis and discitis (Figure 2c). Cauda equina syndrome developed with progressive lower limb weakness and hypoaesthesia. A 3-month course of antibiotic therapy was necessary. The final VA was 6/12 right and 6/6 left, and full leg mobility was regained.

\section{Case 3}

A 62-year-old female was admitted to intensive care with septic shock having been found unconscious at home. She had undergone lumbar laminectomy surgery 6-weeks earlier. MRI showed lumbar spine osteomyelitis. Her left eye had total hypopyon with marked vitreous opacification on ultrasound scan. Intravitreal ceftazidime $(2 \mathrm{mg} / 0.1 \mathrm{ml})$ and vancomycin $(1 \mathrm{mg} / 0.1 \mathrm{ml})$ were administered. MSSA was identified from blood, vitreous and spinal wound cultures. Despite intensive management the patient died three days later.

\section{Discussion}

To our knowledge this is the first series describing an association between Staphylococcal vertebral osteomyelitis and EBE. Pyogenic vertebral osteomyelitis (PVO) may cause severe local effects, including cord compression, and systemic effects related to bacteraemia and disseminated infection. The leading cause of PVO is S.aureus, ${ }^{6,7}$ and the incidence of PVO is rising, particularly in the elderly. ${ }^{7}$

Up to $25 \%$ of EBE cases are initially misdiagnosed, ${ }^{2}$ and PVO diagnosis is often delayed. ${ }^{7}$ Early recognition of EBE associated with PVO may therefore be challenging. In cases 1 and 2, a link between back pain and ocular presentation was not initially recognised. Subsequently, both patients developed sepsis. ${ }^{8}$ In case 2 , sightthreatening ocular disease and disseminated infection was evident at presentation to our unit. Despite delays in initiation of antibiotic therapy both patients achieved excellent visual outcomes and osteomyelitis was managed conservatively with antibiotic therapy.

Many patients with EBE have underlying disease, such as diabetes, predisposing to infection. ${ }^{2}$ Cases 1 and 2 are unusual with no predisposing conditions. In case 3 , infection was a complication of surgery and she may have been immunocompromised following previous intravenous drug abuse.

Prompt identification of the responsible organism and any foci of infection are paramount in EBE. Blood, urine and stool cultures and inflammatory markers are important. Further sampling and imaging of any suspected tissue/organs is required. Urgent intraocular sampling is necessary if the responsible organism is not identified and should precede administration of systemic antibiotics. Despite substantial risks to both vision and 
life, prompt investigation and treatment can achieve favourable visual and systemic outcomes.

\section{Summary}

What was known before

- Bacterial endogenous endophthalmitis is a sightthreatening ophthalmic emergency.

- The condition should be suspected in any septic patient developing eye symptoms.

- Common primary sources of infections are endocardium, liver, lung, urinary tract, and meninges.

\section{What this study adds}

- Spinal bone infections are a rare source of infection in endogenous endophthalmitis.

- Osteomyelitis should be considered as an infective source in any such patient reporting bone pain.

- Pyogenic vertebral infections are typically a disease of older people ( $>60$ years) and should particularly be considered in older patients with endogenous endophthalmitis; in this group S. aureus is a common organism.

\section{Conflict of interest}

The authors declare no conflict of interest.

\section{References}

1 Okada AA, Johnson RP, Liles WC, D'Amico DJ, Baker AS. Endogenous bacterial endophthalmitis. Report of a ten-year retrospective study. Ophthalmology 1994; 101(5): 832-838.

2 Jackson TL, Paraskevopoulos T, Georgalas I. Systematic review of 342 cases of endogenous bacterial endophthalmitis. Surv Ophthalmol 2014; 59(6): 627-635.

3 Lee SY, Chee Sp. Group B Streptococcus endogenous endophthalmitis: case reports and review of the literature. Ophthalmology 2002; 109: 1879-1886.

4 Smith JM, Griffiths PG, Fraser SG. Acute red eye and back pain as a presentation for systemic illness: case report. BMC Ophthalmol 2006; 6: 31.

5 Christensen SR, Hansen AB, La Cour M, Fledelius HC. Bilateral endogenous bacterial endophthalmitis: a report of four cases. Acta Ophthalmol Scand 2004; 82: 306-310.

6 Jeong SJ, Choi SW, Youm JY, Kim HW, Ha HG, Yi JS. Microbiology and epidemiology of infectious spinal disease. J Korean Neurosurg Soc 2014; 56: 21-27.

7 Gupta A, Kowalski TJ, Osmon DR, Enzler M, Steckelberg JM, Huddleston PM et al. Long-term outcome of pyogenic vertebral osteomyelitis: a cohort study of 260 patients. Open Forum Infect Dis 2014; 1(3): ofu107.

8 Bone RC, Balk RA, Cerra FB, Dellinger RP, Fein AM, Knaus WA et al. Definitions for sepsis and organ failure and guidelines for the use of innovative therapies in sepsis. The American College of Chest Physicians/Society of Critical Care Medicine Consensus Conference Committee. Chest 1992; 101: 1644-1655. 